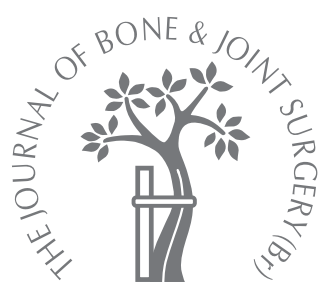

\title{
Internal rotational error of the tibial component is a major cause of pain after total knee replacement
}

\author{
D. Nicoll, \\ D. I. Rowley \\ From the University \\ of Dundee, Dundee, \\ United Kingdom
}

D. Nicoll,

FRCSEd(Trauma\&Orth),

Clinical Lecturer \& Specialist

Registrar

D. I. Rowley, BMedBiol,

FRCS, MD, Emeritus Professor

\& Consultant Orthopaedic

Surgeon

Department of Orthopaedic \&

Trauma Surgery

Ninewells Hospital \& Medical

School, Dundee DD1 9SY, UK.

Correspondence should be sent to $\mathrm{Mr}$ D. Nicoll; e-mail:

nicoll_dave@yahoo.co.uk

(C)2010 British Editorial Society of Bone and Joint Surgery doi:10.1302/0301-620X.92B9. $23516 \$ 2.00$

$J$ Bone Joint Surg [Br] 2010;92-B:1238-44. Received 26 February 2010; Accepted after revision 15 April 2010

This study used CT analysis to determine the rotational alignment of 39 painful and 26 painless fixed-bearing total knee replacements (TKRs) from a cohort of 740 NexGen Legacy posterior-stabilised and cruciate-retaining prostheses implanted between May 1996 and August 2003.

The mean rotation of the tibial component was $4.3^{\circ}$ of internal rotation $\left(25.4^{\circ}\right.$ internal to $13.9^{\circ}$ external rotation) in the painful group and $2.2^{\circ}$ of external rotation $\left(8.5^{\circ}\right.$ internal to $18.2^{\circ}$ external rotation) in the painfree group $(p=0.024)$. In the painful group 17 tibial components were internally rotated more than $9^{\circ}$ compared with none in the painfree group ( $p<0.001$ ). Additionally, six femoral components in the painful group were internally rotated more than $6^{\circ}$ compared with none in the painfree group $(p=0.017)$. External rotational errors were not found to be associated with pain.

Overall, 22 (56.4\%) of the painful TKRs had internal rotational errors involving the femoral, the tibial or both components. It is estimated that at least $4.6 \%$ of all our TKRs have been implanted with significant internal rotational errors.

Total knee replacement (TKR) is one of the most successful orthopaedic procedures with satisfaction rates of over $90 \% .{ }^{1}$ However, the incidence of dissatisfaction and moderate pain after TKR is about $13 \%$ at one year ${ }^{2,3}$ and $20.5 \%$ at two to seven years after surgery. ${ }^{4}$

Many intra- and extra-articular causes of pain after TKR have been identified or proposed, ${ }^{5-7}$ but often no cause can be found. In recent years internal rotational malalignment of components has been implicated as a cause of pain after TKR. ${ }^{8,9}$

The incidence of rotational errors in TKR with unexplained pain has not been reported previously. Furthermore, while internal rotation of more than $6^{\circ}$ of the femoral component has been associated with pain ${ }^{8,9}$ the amount of internal rotation of the tibial component required to produce pain is not known.

Our aim was to identify the incidence of rotational errors in painful and painfree TKR and to explore the relationship between component rotation and pain after TKR.

\section{Patients and Methods}

Between May 1996 and August 2003, we performed 740 NexGen Legacy posterior-stabilised and NexGen Legacy cruciate-retaining (Zimmer Inc., Warsaw, Indiana) fixed-bearing TKRs. All were carried out without navigation using a measured resection technique after ligament balancing with intramedullary alignment of the femoral component and extramedullary alignment of the tibial component. Intra-operatively, rotation of the femoral component was referenced from the posterior condylar axis or the transepicondylar axis. Rotation of the tibial component was referenced from the medial third of the tibial tuberosity. All were reviewed at one year and every two years thereafter by recording the Knee Society score (KSS). ${ }^{10}$ A total of 60 patients (61 TKRs) with unexplained moderate pain were identified at least one year after surgery. Moderate knee pain was defined as a KSS of $>20$ points at any review. All the patients had been seen by their operating surgeon and no identifiable cause of their pain had been found. Infection had been excluded either by the presence of a normal value for serum CRP with no clinical suspicion of infection or by a sterile knee aspirate. No TKRs in the study had clinical evidence of instability.

Of these 60 patients, three had died, one had moved abroad and two were excluded since they had sustained periprosthetic fractures. After obtaining ethical approval for the study, the remaining 54 were invited to attend for CT scans to determine the rotational alignment of the tibial and femoral components according 


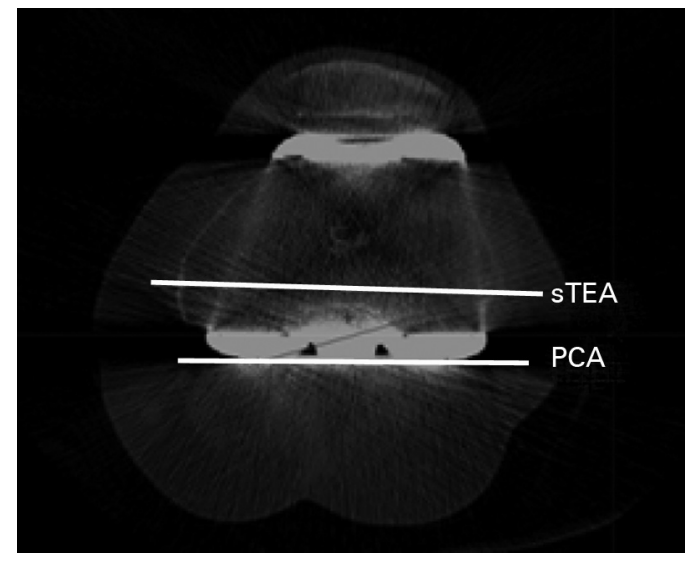

Fig. 1

CT scan showing that rotation of the femoral component was calculated from the angle formed between the posterior condylar axis (PCA) and the surgical transepicondylar axis (sTEA).

to the technique of Berger et al. ${ }^{11}$ Six patients declined to participate because of ill health, eight failed to respond and one refused to undergo CT. One patient was later excluded because of deep tissue-culture evidence of prosthetic infection at revision surgery. This left 38 patients (39 TKRs) who attended for CT analysis.

The senior author (DN) was present when each CT was performed to ensure that the Berger protocol was strictly followed. Rotation of the femoral component was referenced from an axial slice through the distal femoral epicondyles from the angle formed by a line along the posterior condylar axis of the femoral component and a line connecting the sulcus of the medial epicondyle and the most prominent part of the lateral epicondyle (the surgical transepicondylar axis, Fig. 1). Neutral rotation was considered to be $3.5^{\circ}$ of internal rotation in men and $0.3^{\circ}$ of internal rotation in women ${ }^{11}$ from the surgical transepicondylar axis. Rotation of the tibial component was determined from three axial slices, one just below the tibial base plate, one at the level of the polyethylene tibial insert and one at the level of the most prominent part of the tibial tuberosity (Fig. 2). The tibial tuberosity axis was plotted from a line connecting the geometrical centre of an ellipse of best fit around the proximal tibia just below the metal base plate which was transposed to the image at the level of the tuberosity and the centre of the most prominent part of the tibial tuberosity. Rotation of the tibial components was determined by the angle formed perpendicular from the posterior edge of the polyethylene insert (the tibial component axis) which was transposed to the image at the level of the tuberosity and the tibial tuberosity axis (Fig. 2). Neutral rotation of the tibial component was considered to be $18^{\circ}$ of internal rotation from the centre of the tip of the tuberosity for both genders which corresponds to the rotation of the normal native articular surface. ${ }^{12}$
All the high-quality digital CT scans were analysed by the senior author with anatomical and component landmarks plotted to a single pixel and angles measured using a programme designed by our Department of Applied Computing for use with MATLAB version 6.5 software (The MathWorks Inc., Natick, Massachussets).

Rotational alignments of our patients were compared with those of a painfree control group of patients who had never reported pain in their knee following their TKR, which had been performed at least one year previously. After a provisional analysis of rotation of the tibial component of the painful group a power calculation was performed based on the finding of an incidence of $41 \%$ of internal rotation of the component of more than $10^{\circ}$ in the painful group and a postulated incidence of $0 \%$ in the painfree control group. With a power level set at 0.90 and the probability of a type-I error set at 0.01 it was calculated that 26 TKRs would be required for the painless control group for comparison. Accordingly, we selected 26 painless TKRs at random in 25 patients as the control group. They underwent radiological and CT analysis, and clinical assessment. Once all the data had been collected for both groups, all the rotational measurements for the components for both groups were measured on digital images by the senior author in a blinded manner, which was repeated with a minimum of three months between measurements. Final values for the rotational alignment of both components were calculated as the mean of two measures and test re-test reliability was assessed.

Internal rotation of the component was considered to be a positive value and external rotation, a negative value. Neutral rotation of $0^{\circ}$ was determined at the point of $18^{\circ}$ of internal rotation from the tip of the tibial tuberosity. ${ }^{12}$ In addition to measuring isolated rotation of the femoral and tibial component, other rotational parameters measured included rotational mismatch (tibial component rotation femoral component rotation) and combined rotation (tibial component rotation + femoral component rotation) (Fig. 3). In keeping with findings from previous studies, the femoral components were considered to be excessively rotated if they were internally rotated more than $6^{\circ 8,9}$ or externally rotated $8^{\circ} .{ }^{13}$ Values for excessive malrotation of the tibial component were to be explored in the analysis of the data.

When they came for their CT scan, all patients completed a visual analogue scale (VAS) for the pain (0 for no pain, 10 for severe pain) in their TKR on an average day and their knee was examined to calculate a KSS. Alignment of the components was also assessed from post-operative digital radiographs using a computerised system developed in our department ${ }^{14}$ which referenced both coronal and sagittal alignment from the anatomical axes of the femur and tibia in both planes. The mean values for alignment were compared and outliers in the coronal plane were identified. An outlier was defined as being more than $3^{\circ}$ outside the recommended alignment for that parameter. Recommended alignment was considered to be $6^{\circ}$ of valgus for tibiofemoral 


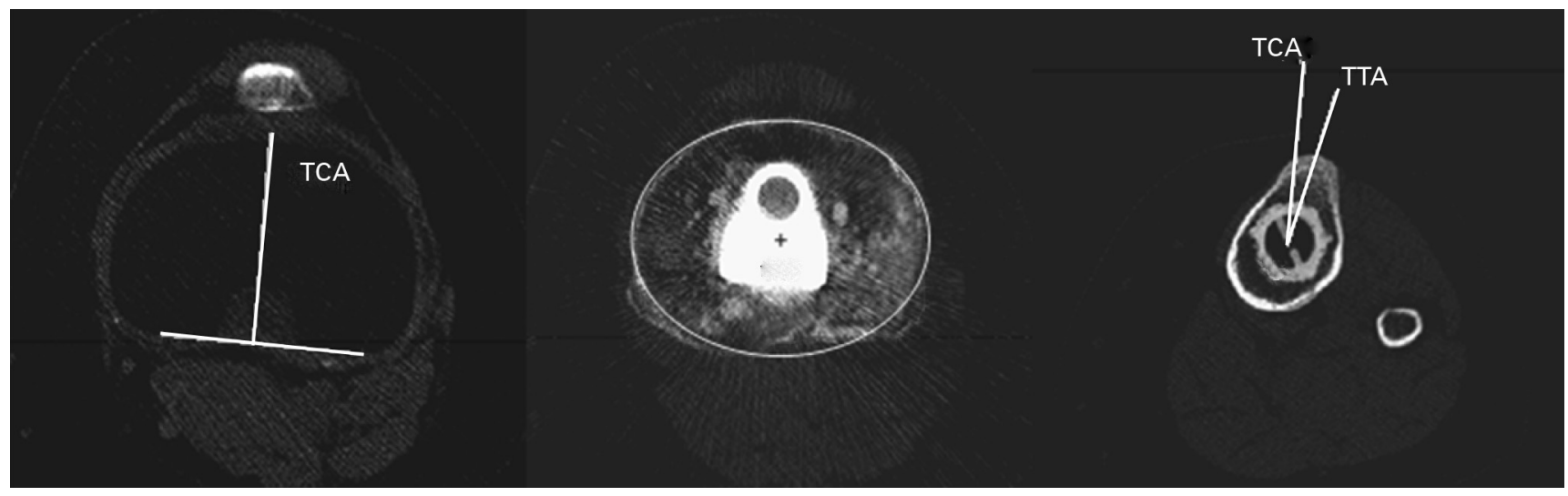

Fig. 2

CT scans showing that rotation of the tibial component was calculated from the angle formed by the tibial tuberosity axis (TTA) and the tibial condylar axis (TCA).
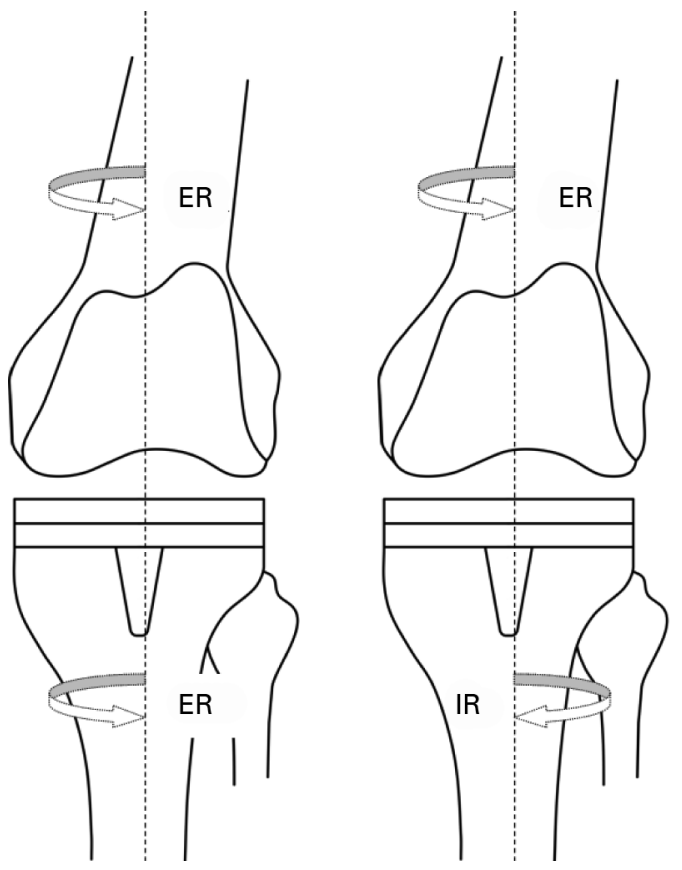
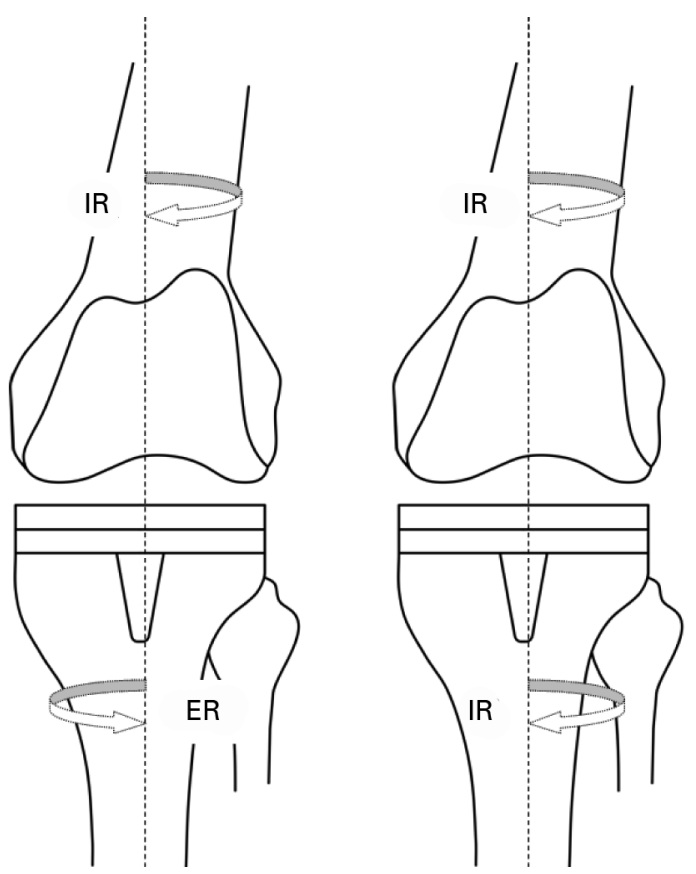

Fig. 3

Diagrams of varying configurations of malrotation showing a) combined component ER, b) tibial component IR mismatch, c) tibial component ER mismatch, and d) combined component IR (ER, external rotation; IR, internal rotation).

coronal alignment, $6^{\circ}$ of valgus for coronal alignment of the femoral component, $0^{\circ}$ for coronal alignment of the tibial component, $0^{\circ}$ for sagittal alignment of the femoral component and a posterior slope of $7^{\circ}$ of the tibial component.

Statistical analysis. Data were checked for normality of distribution using the Kolmogorov-Smirnov test. For normally distributed data Student's $t$-test was used to compare two groups and when data were not normally distributed the Mann-Whitney U test was applied. Fisher's exact test was used to compare frequencies between groups. Test re-test reliability of femoral and tibial rotation was determined using Pearson's correlation coefficient to identify the mean difference between repeated measures. The level of statistical significance was set at a $\mathrm{p}$-value $\leq 0.05$.

\section{Results}

Details of the groups are shown in Table I. They were similar in terms of clinical details, the type of implant, preoperative deformity and the incidence of pre-operative patellofemoral pain and of patellar resurfacing. The mean KSS was lower in the painful group by 40.6 points (MannWhitney $U$ test, $\mathrm{p}=0.001$ ) primarily because pain is so heavily weighted in this system. The mean total function component of the KSS was lower in the painful group by 
Table I. Clinical details (mean, SD) of the study groups

\begin{tabular}{|c|c|c|c|}
\hline & Painful group & Painless group & p-value \\
\hline Number of patients & 38 (39 TKRs*) & 25 (26 TKRs) & - \\
\hline Age (yrs) & $68.5(10.5)$ & $65.6(10.9)$ & $0.29^{\dagger}$ \\
\hline Male:female & $16: 22$ & $14: 12$ & $0.50^{\ddagger}$ \\
\hline Body mass index in $\mathrm{kg} / \mathrm{m}^{2}$ & $29.4(4.0)$ & $28.5(4.7)$ & $0.41^{\dagger}$ \\
\hline$L: R$ & $16: 23$ & $11: 15$ & $0.99^{\ddagger}$ \\
\hline Pre-operative varus:valgus & $35: 4$ & $22: 4$ & $0.70^{\ddagger}$ \\
\hline Pre-operative fixed flexion $\geq 10^{\circ}$ & $21: 18$ & $15: 11$ & $0.80^{\ddagger}$ \\
\hline Mean time since surgery in years & $5.2(2.3)$ & $4.2(1.7)$ & $0.08^{\dagger}$ \\
\hline \multicolumn{4}{|l|}{ Implant type $\mathrm{s}^{\S}$} \\
\hline PS:CR & $33: 6$ & $24: 2$ & $0.46^{\ddagger}$ \\
\hline Pre-operative patellofemoral pain & 19 & 17 & $0.84^{\ddagger}$ \\
\hline Patella resurfaced & 22 & 13 & $0.80^{\pi}$ \\
\hline Mean Knee Society score & $53.6(17.3)$ & $94.2(6.1)$ & $0.001^{\pi}$ \\
\hline Mean function score & $50.8(16.8)$ & $81.0(20.6)$ & $0.029^{\pi}$ \\
\hline Mean pain score & $11.6(15.5)$ & $50.0(0)$ & $<0.001^{\pi}$ \\
\hline Mean visual analogue scale for pain & $5.4(1.3)$ & $0.1(0.1)$ & $<0.001^{\Uparrow}$ \\
\hline \multicolumn{4}{|l|}{ * TKR, total knee replacement } \\
\hline \multicolumn{4}{|l|}{$\dagger t$-test } \\
\hline \multicolumn{4}{|l|}{$\ddagger$ Fisher's exact test } \\
\hline \multicolumn{4}{|c|}{ § PS, posterior-stabilised; CR, cruciate-retaining } \\
\hline \multicolumn{4}{|c|}{ II Mann-Whitney U test } \\
\hline
\end{tabular}

Table II. Details (mm, SD) coronal and sagittal plane alignments in both groups

\begin{tabular}{|c|c|c|c|}
\hline & Painful ( $n=39$ ) & Painless $(n=26)$ & p-value \\
\hline Coronal tibiofemoral alignment & $5.7^{\circ}$ of valgus $\left(2.9^{\circ}\right)$ & $6.3^{\circ}$ of valgus $\left(2.3^{\circ}\right)$ & $0.41^{*}$ \\
\hline Outliers & 9 & 4 & $0.54^{\dagger}$ \\
\hline Coronal femoral component alignment & $6.6^{\circ}$ of valgus $\left(2.7^{\circ}\right)$ & $6.6^{\circ}$ of valgus $\left(2.0^{\circ}\right)$ & $0.92^{*}$ \\
\hline Outliers & 4 & 2 & $1.00^{\dagger}$ \\
\hline Coronal tibial component alignment & $0.4^{\circ}$ of varus $\left(2.5^{\circ}\right)$ & $0.2^{\circ}$ of varus $\left(2.7^{\circ}\right)$ & $0.75^{*}$ \\
\hline Outliers & 5 & 4 & $1.00^{\dagger}$ \\
\hline Mean femoral component sagittal alignment & $0.2^{\circ}$ of flexion $\left(3.0^{\circ}\right)$ & $0.4^{\circ}$ of flexion $\left(3.3^{\circ}\right)$ & $0.79^{*}$ \\
\hline Mean tibial component sagittal alignment & $7.3^{\circ}$ of posterior slope $\left(2.5^{\circ}\right)$ & $7.2^{\circ}$ of posterior slope $\left(2.3^{\circ}\right)$ & $0.85^{*}$ \\
\hline
\end{tabular}

30.2 points (Mann-Whitney U test, $\mathrm{p}=0.029$ ) (Table I). No TKR in the painful group had a VAS for pain of less than 4.0 while in the painless group no VAS for pain was greater than 0.5 (Table I). The groups were similar with regard to coronal tibiofemoral and individual component alignment in both the coronal and sagittal planes (Table II).

A summary of the rotation alignments for each group is given in Table III.

Rotation of the femoral component. Pearson's correlation coefficient for repeated measures of rotation of the femoral component was 0.81 , demonstrating very strong correlation, with a mean difference of $1.03^{\circ}$. While there was no significant difference in the mean rotation between the groups (Table II), in the painful group eight femoral components were internally rotated more than $6^{\circ}$ compared with none in the painfree group (student's t-test, $p=0.017)$. The size of internal rotational errors was small with the maximum internal rotational error encountered being $8.8^{\circ}$. No femoral component in either group was excessively externally rotated.

Rotation of the tibial component. Pearson's correlation coefficient for repeated measures of rotation of the tibial component was 0.87 , demonstrating very strong correlation, with a mean difference of $0.83^{\circ}$. It was much more variable than rotation of the femoral component particularly in the painful group with a range of rotation of $43.6^{\circ}$ between the most internally rotated and most externally rotated tibial components. In the painful group rotation of the tibial component 
Table III. Details of rotational alignment of total knee replacement (TKR) components for both groups

\begin{tabular}{|c|c|c|c|}
\hline & Painful group & Painfree group & p-value \\
\hline \multicolumn{4}{|l|}{ Femoral component } \\
\hline Mean $\left(\right.$ range $^{\circ}$ ) femoral component rotation & $2.0 \mathrm{IR}(8.8 \mathrm{IR} \text { to } 3.9 \mathrm{ER})^{\dagger}$ & $0.8 \mathrm{IR}$ (5.9 IR to $6.8 \mathrm{ER})$ & $0.17^{*}$ \\
\hline Number of femoral components internally rotated $>6^{\circ}$ & 8 & 0 & $0.017^{\pi}$ \\
\hline Number of femoral components externally rotated $>8^{\circ}$ & 0 & 0 & $1.00^{\pi}$ \\
\hline \multicolumn{4}{|l|}{ Tibial component } \\
\hline Mean (range, ${ }^{\circ}$ ) femoral component rotation & $4.3 \mathrm{IR}$ (25.4 IR to $13.9 \mathrm{ER})$ & 2.2 ER (8.5 IR to $18.2 \mathrm{ER}$ ) & $0.003^{*}$ \\
\hline Number of tibial components internally rotated $>9^{\circ}$ & 17 & 0 & $<0.001^{\pi}$ \\
\hline Number of tibial components externally rotated $>10^{\circ}$ & 2 & 4 & $0.39 \pi$ \\
\hline \multicolumn{4}{|l|}{ Combined rotation ${ }^{\ddagger}$} \\
\hline Mean (range, ${ }^{\circ}$ ) combined component rotation & $8.0 \mathrm{IR}$ (25.6 IR to $22.1 \mathrm{ER})$ & $1.3 \mathrm{ER}$ (10.7 IR to $14.3 \mathrm{ER})$ & $<0.001^{*}$ \\
\hline Number of TKRs in combined component IR $>11^{\circ}$ & 15 & 0 & $<0.001^{\uparrow}$ \\
\hline \multicolumn{4}{|l|}{ Rotational mismatch ${ }^{\S}$} \\
\hline Mean $\left(\right.$ range $^{\circ}$ ) tibial component rotational mismatch & $2.6 \mathrm{IR}$ (25.6 IR to $21.1 \mathrm{ER}$ ) & 3.1 ER (10.3 IR to $22.1 \mathrm{ER})$ & $0.025^{*}$ \\
\hline Number of TKRs with tibial component IR mismatch $>11^{\circ}$ & 11 & 0 & $0.002^{\pi}$ \\
\hline Number of TKRs with tibial component ER mismatch $>10^{\circ}$ & 8 & 6 & $1.00^{* *}$ \\
\hline \multicolumn{4}{|l|}{$* t$-test } \\
\hline \multicolumn{4}{|l|}{$\dagger I R$, internal rotation; ER, external rotation } \\
\hline \multicolumn{4}{|l|}{ ‡ tibial component rotation + femoral component rotation } \\
\hline \multicolumn{4}{|l|}{$\S$ tibial component rotation - femoral component rotation } \\
\hline I Fisher's exact test & & & \\
\hline
\end{tabular}

was a mean of $6.5^{\circ}$ greater than that in the painfree group (Student's $t$-test, $\mathrm{p}=0.003$, Table III). From the data it appeared that internal rotation of more than $9^{\circ}$ was a significant cut-off value associated with pain. In all, 17 tibial components were internally rotated more than $9^{\circ}$ in the painful group while no tibial component was internally rotated more than $9^{\circ}$ in the painfree group (Fisher's exact test, $\mathrm{p}<0.001$ ).

Excessive external rotation of the tibial component did not appear to be associated with pain. There was a greater proportion of externally rotated tibial components in the painfree group, but the difference was not statistically significant (Table III). External rotation of the tibial component of up to $18.2^{\circ}$ was tolerated in one patient in the painfree group.

Excessive internal rotation of either or both TKR components. When considering femoral internal rotation of more than $6^{\circ}$ and internal rotation of the tibial component of more than $9^{\circ}$ to be excessive, 22 of the 39 painful TKRs $(56.4 \%)$ had excessive internal rotation of either the femoral component, the tibial component or both compared with none in the painfree group (Student's $t$-test, $\mathrm{p}<0.001$, Table III). In all, five painful TKRs had isolated excessive internal rotation of the femoral component while 14 had isolated excessive internal rotation of the tibial component. In three painful TKRs excessive internal rotation was found in both components. Based on an incidence of moderate pain after TKR of $8.2 \%$ and a finding of 22 knees $(56.4 \%)$ with significant internal rotation of the components in our group of 39 knees (38 patients), we have estimated an incidence of significant internal rotation errors of $4.6 \%$ of all our TKRs.
Combined rotation of the components. In the painful group the components were implanted with a mean of $8.0^{\circ}\left(25.6^{\circ}\right.$ internal to $22.1^{\circ}$ external rotation) of combined internal rotation while in the painfree group the components were implanted with a mean of $1.3^{\circ}\left(10.7^{\circ}\right.$ internal to $14.3^{\circ}$ external rotation) of combined external rotation (Student's $t$-test, $\mathrm{p}<0.001$, Table III). In the painful group 15 TKRs were in $>11^{\circ}$ of combined internal rotation compared with none in the painfree group (Fisher's exact test, $\mathrm{p}<0.001$ ). All 15 TKRs with $>11^{\circ}$ of combined internal rotation had more than $9^{\circ}$ of internal rotation of their tibial component. Rotational mismatch between the tibial and femoral components. This was subject to great variation especially in the painful group. There was a significant difference in the mean rotational mismatch with TKRs in the painful group having a mean of $2.6^{\circ}\left(25.6^{\circ}\right.$ internal to $21.1^{\circ}$ external rotation) of internal rotational mismatch of the tibial component while the painfree group had a mean of $3.1^{\circ}\left(10.3^{\circ}\right.$ internal to $21.1^{\circ}$ external rotation) of external rotational mismatch of the tibial component (Student's $t$-test, $\mathrm{p}=0.025$ ) (Table III). A total of 11 of the TKRs in the painful group had an internal rotational mismatch of the tibial component of more than $11^{\circ} \mathrm{com}-$ pared with none in the painfree group (Fisher's exact $t$ test, $p=0.002)$. However, all of the 11 TKRs with internal rotational mismatch of the tibial component of more than $11^{\circ}$ had internal rotation of the tibial component greater than $9^{\circ}$. Up to $22.1^{\circ}$ of external rotational of the tibial component mismatch was tolerated in the painfree group. 


\section{Discussion}

We have identified an incidence of $8.2 \%$ of moderate pain at a minimum of one year after TKR. Although this value may appear to be surprising, it is less than in some other studies. 3,4 This may reflect differences in the definition of 'moderate' pain. We have attempted to establish how many TKRs with unexplained pain have malrotation of one or both components when assessed by CT.

We found that more than half of the painful TKRs in our study group had internal rotational errors in the alignment of TKR components, particularly the tibial implant. The incidence of excessive internal rotation of the tibial component was more than double that of excessive internal rotation of the femoral component and the size of internal rotational errors of the tibial component was much greater than that for the femoral component. Additionally, while we have demonstrated a strong association between excessive internal rotational errors and pain, external rotational errors of the femoral component were not detected and quite large values of external rotation of the tibial component were also tolerated in the painfree group.

From our data, we have estimated an incidence of $4.6 \%$ of internal rotational error. This may be an under-estimate since it is not known if excessive internal rotation is also present in patients with only mild pain after TKR.

The findings of our study support previous work by Barrack et $\mathrm{al}^{8}$ which also identified internal rotation of the tibial component as a cause of pain after TKR. Our mean value for internal rotation of $4.2 \%$ of the tibial component is similar to their finding of $6.2^{\circ}$ in TKRs with anterior knee pain. However, their study focused solely on TKRs with anterior knee pain rather than all painful TKRs and made no comment on the frequency of rotational errors or the extent of internal rotation required to produce symptoms.

In our study we have identified a threshold value of $9^{\circ}$ of internal rotation of the tibial component, representing a total of $27^{\circ}$ of internal rotation from the tip of the tibial tuberosity, as that required to produce pain, with internal rotation of the tibial component of up to $8^{\circ}$ being tolerated in the painfree group.

The variation in rotation of the tibial component and the size of internal rotational errors in our study was surprisingly large with a difference of $43.6^{\circ}$ between the most internally rotated and the most externally rotated tibial component. The maximum internal rotational error found was $25.4^{\circ}$. Our findings are similar to those of a previous study by Matsuda et $\mathrm{al}^{15}$ which identified internal rotational errors of the tibial component of up to $28^{\circ}$. By contrast, rotational errors in the placement of the femoral component in our study occurred less frequently and were small. Again this is comparable with previous data on rotation of the femoral component without navigation. ${ }^{16}$

It remains unclear why internal rotational errors cause pain after TKR. Internal rotation of the tibial component results in a relative external rotation of the tibia. It has been postulated that the resultant increase in the $\mathrm{Q}$ angle places abnormal stresses on the extensor mechanism. ${ }^{8}$ Inadvertent internal rotation of the tibial component can occur for a number of reasons. The extensor mechanism and lateral soft tissues can deflect the final tibial cutting jig into internal rotational particularly in a tight knee or in an obese patient. Additionally, when implanting a symmetrical tibial component an apparently appropriately-sized implant if normally orientated may overhang posterolaterally, a situation which has been implicated as causing pain by impingement on the popliteus tendon. ${ }^{5}$ In attempting to avoid this the surgeon may internally rotate the tibial component to obtain better cover of the cut tibial surface. A preferable option would be to use a smaller tibial component allowing correct rotational alignment without posterolateral overhang. It also remains unclear why apparently extensive external rotation of the tibial component can be tolerated without pain.

While combined component internal rotation of greater than $11^{\circ}$ and internal rotational mismatch of the tibial component alone of greater than $11^{\circ}$ may be associated with pain, all TKRs with excessive combined internal rotation and excessive mismatch of tibial internal rotation had more than $9^{\circ}$ of internal rotation of the tibial component. It therefore remains unclear if either combined component rotation or tibial internal rotational mismatch can cause pain with less than $9^{\circ}$ of internal rotation of the tibial component.

Our study was confined to fixed-bearing implants. It remains uncertain if mobile-bearing TKRs would be more tolerant of internal rotational errors since the polyethylene bearing in mobile-bearing knees has been found to rotate up to $10^{\circ}$ on the tibial tray in vivo. ${ }^{17,18}$ This might compensate for some degree of internal rotational error. However, further studies have shown no improvement in patellar tracking $^{19}$ in mobile-bearing TKRs as compared with fixedbearing implants with a similar degree of tibiofemoral rotation throughout the gait cycle in both designs. ${ }^{20}$ Furthermore, randomised, controlled trials ${ }^{21,22}$ and large meta-analysis ${ }^{23}$ have shown no clinical advantage of mobile-bearing TKRs over fixed-bearing designs.

There is also conflicting evidence as to whether computer navigation improves the accuracy of component rotation. While image-free navigation has not been found to improve rotational accuracy, ${ }^{24}$ another study comparing CT-based navigation and conventional techniques has identified a reduction in the variation of rotation of both components with CT-based navigation and a reduction in the number of outliers with rotational values outside a recommended reference range of $\mathrm{SD} 3^{\circ} .^{16}$

In summary, we found that internal rotational errors, particularly of the tibial component, were a major cause of pain and functional deficit after TKR. Great care should be taken when establishing rotational alignment of the tibial component with careful reference to the tibial tuberosity.

Our study was supported by a grant of $£ 15000$ from the Chief Scientist Office of the Scottish Government Health Directorate. Thanks are given to Dr M. Seise of the School of Computing, University of Dundee, for assistance with analysis of the CT scans and to Dr T. B. Oliver of the Department of Radiology, Ninewells Hospital and Medical School, Dundee, for assistance with the CT. 
No benefits in any form have been received or will be received from a commercial party related directly or indirectly to the subject of this article.

\section{References}

1. Robertsson 0, Dunbar M, Pehrsson T, Knutson K, Lidgren L. Patient satisfaction after knee arthroplasty: a report on 27,372 knees operated on between 1981 and 1995 in Sweden. Acta Orthop Scand 2000;71:262-67.

2. Brander VA, Stulberg SD, Adams AD, et al. Predicting total knee replacement pain: a prospective, observational study. Clin Orthop 2003;416:27-36.

3. Baker PN, van der Meulen JH, Lewsey J, et al. The role of pain and function in determining patient satisfaction after total knee replacement: data from the National Joint Registry for England and Wales. J Bone Joint Surg [Br] 2007;89B:893-900.

4. Hawker G, Wright J, Coyte P, et al. Health-related quality of life after knee replacement. J Bone Joint Surg [Am] 1998;80-A:163-73.

5. Dennis DA. Evaluation of painful total knee arthroplasty. J Arthroplasty 2004; 19:35-40.

6. Thornhill TS. Painful total knee arthroplasty. Orthopaedics 2002;25:965-7.

7. Toms AD, Mandalia V, Haigh R, Hopwood B. The management of patients with painful total knee replacement. J Bone Joint Surg [Br] 2009;91-B:143-50.

8. Barrack RL, Schrader T, Bertot AJ, Wolfe MW, Myers L. Component rotation and anterior knee pain after total knee arthroplasty. Clin Orthop 2001;392:46-55.

9. Hofmann S, Romero J, Roth-SchiffI E, Albrecht T. Rotational malalignment of the components may cause chronic pain or early failure in total knee arthroplasty. Orthopade 2003;32:469-76 (in German).

10. Insall JN, Dorr LD, Scott RD, Scott WN. Rationale of the Knee Society clinical rating system. Clin Orthop 1989;248:13-14.

11. Berger RA, Crossett LS, Jacobs JJ, Rubash HE. Malrotation causing patellofemoral complications after total knee arthroplasty. Clin Orthop 1998;356:14453.

12. Berger RA, Seel MJ, Schleiden M, et al. Computerized tomographic determination of the normal tibiofemoral rotational angles: a guide to tibial component rotational alignment in TKA. Orthop Trans 1993;17:1174.
13. Nagamine R, White SE, McCarthy DS, Whiteside LA. Effect of rotational malposition of the femoral component on knee stability kinematics after total knee arthroplasty. J Arthroplasty 1995;10:265-70.

14. Prakash U, Wigderowitz CA, McGurty DW, Rowley DI. Computerised measurement of tibiofemoral alignment. J Bone Joint Surg [Br] 2001;83-B:819-24.

15. Matsuda S, Miura H, Nagamine R, et al. Effect of femoral and tibial component position on patellar tracking following total knee arthroplasty: 10-year follow-up of Miller-Galante I knees. Am J Knee Surg 2001;14:152-6.

16. Mizu-uchi H, Matsuda S, Miura H, et al. The evaluation of post-operative alignment in total knee replacement using a CT-based navigation system. J Bone Joint Surg [Br] 2008;90-B:1025-31.

17. Komistek RD, Dennis DA, Mahfouz MR, Walker S, Outten J. In vivo polyethylene bearing mobility is maintained in posterior stabilized total knee arthroplasty. Clin Orthop 2004:428:207-13.

18. Dennis DA, Komistek RD. Kinematics of mobile-bearing total knee arthroplasty. Instr Course Lect 2005;54:207-20.

19. Pagnano MW, Trousdale RT, Stuart MJ, Hanssen AD, Jacofsky DJ. Rotating platform knees did not improve patellar tracking: a prospective, randomized study of 240 primary total knee arthroplasties. Clin Orthop 2004;428:221-7.

20. Dennis DA, Komistek RD, Mahfouz MR, Walker SA, Tucker A. A multicenter analysis of axial femorotibial rotation after total knee arthroplasty. Clin Orthop 2004;428:180-9.

21. Harrington MA, Hopkinson WJ, Hsu P, Manion L. Fixed- vs mobile-bearing total knee arthroplasty: does it make a difference?: a prospective randomized study. $J$ Arthroplasty 2009;24(Suppl):24-7.

22. Wylde V, Learmouth I, Potter A, Bettinson K, Lingard E. Patient-reported out comes after fixed- versus mobile-bearing total knee replacement: a multi-centre randomised controlled trial using the Kinemax total knee replacement. J Bone Joint Surg [Br] 2008;90-B:1172-9.

23. Oh KJ, Pandher DS, Lee SH, Sung Joon SD, Lee ST. Meta-analysis comparing outcomes of fixed-bearing and mobile-bearing prostheses in total knee arthroplasty. J Arthroplasty 2009:24:873-84.

24. Kim YH, Kim JS, Choi Y, Kwon OR. Computer-assisted navigation does not improve the alignment and orientation of the components in total knee arthroplasty. J Bone Joint Surg [Am] 2009;91-A:14-19. 